\title{
Un asunto menor, administrativo: la universidad y el management
}

Por Alejandro Varas, Magister (c) en Filosofia, Pontificia Universidad Católica de Valparaíso

Profesor de Psicología, Pontificia Universidad Católica de Valparaíso

Marcela Mandiola ', Ph.D. en Management, Lancaster University

Profesora de Gestion de Personas en Organizaciones, Universidad Alberto Hurtado

La universidad, su práctica y sus objetivos, han estado en la discusión pública desde hace años. En este artículo reflexionamos acerca de dos discursos principales que han jugado un rol central en la articulación de lo que hoy conocemos como academia en Chile. Lo masculino y managerial le han dado un carácter particular a la universidad en Chile. En otras palabras, la universidad se ha constituido desde un quehacer productivista que busca su optimización a través de la eficiencia y la eficacia. En ese proceso, su dimensión política ha quedado invisibilizada.

A MODO DE

CONTEXTO: LA

UNIVERSIDAD

DE AYSÉN

\begin{abstract}
a última década de nuestra historia en Chile se ha caracterizado por una visibilización de variadas problemáticas sociales y políticas por parte de la población. Uno de los protagonistas de este ciclo ha sido y sigue siendo el movimiento estudiantil, a través de distintas formas de protesta y organización, demandando una educación sin fines de lucro, pública, gratuita, laica y de calidad. En dicho escenario, el segundo gobierno de Michelle Bachelet propuso una reforma educacional que contempló, para la educación superior, "avanzar en la gratuidad universal y efectiva de la educación superior", y una de las propuestas concretas relativas a fortalecer el papel del Estado en educación fue la promulgación de la ley 20.842 que crea dos nuevas universidades estatales: la Universidad de O'Higgins y la Universidad de Aysén.
\end{abstract}

Sin embargo, el pasado 27 de julio del presente año, el Gobierno, a través de la Ministra de Educación, Adriana Delpiano, decidió destituir a la rectora de dicha casa de estudios, la Dra. Roxana Pey. En contradicción con lo planteado en la reforma, el gobierno toma esta decisión luego de que Pey sugiriera la posibilidad de poner en marcha la gratuidad universal para todas y todos los estudiantes de la Universidad de Aysén, argumentando que era completamente viable con el financiamiento a disposición, y propusiera además un sistema orientado a profundizar la democracia interna de la casa de estudios (CNN Chile, 2016). La ministra alega una falta de alineamiento con el gobierno (Bio-Bio Chile, 2016). Ante esta inesperada reacción, Roxana Pey se negó a abandonar el cargo, planteando que"la ministra de Educación no tiene la atribución para lo que está haciendo" (SoyChile.cl, 2016), y argumentó que su decisión se basa en la defensa de la autonomía de las universidades y su compromiso con el 
espíritu de la reforma educativa, llegando incluso a replicar:"me declaro totalmente alineada" (Radio U de Chile, 2016). El Senado de la Universidad de Chile otorgó pleno respaldo a la posición de Roxana Pey, manifestando que "el principio de autonomía universitaria se ve gravemente amenazado". No obstante, el reciente 11 de agosto, la Contraloría General de la República ratificó la decisión del gobierno (El Mostrador, 2016).

\section{Discursos para la universidad}

Indudablemente este conflicto ha puesto nuevamente en tela de juicio a la política institucional por parte de la sociedad, un ejercicio que ya es constante luego del destape de diversos casos de corrupción entre el Estado y grandes empresas. Sin embargo, la particularidad de este hecho está en que nos permite cuestionar cómo se ha configurado la universidad chilena en términos sociales y políticos a lo largo de nuestra historia (Bravo,1992). Analizándolo de un modo mucho más global, vemos que son al menos tres discursos los que informan la práctica universitaria.

En primer lugar estamos ante la presencia de un discurso conservador que ha sido instalado a lo largo de la historia de la universidad en Chile, principalmente a través de la influencia gravitante de la Iglesia Católica y actualmente de la empresa privada en los últimos años. Desde allí, cualquier asomo de cambio que amenace los paradigmas sostenidos por dichos grupos de poder implica una depuración que, en este caso, se expresa a través de una destitución. Como plantea la misma Roxana Pey:"hay personas muy oscuras operando en la reforma Hay dos almas en el gobierno: quienes buscan desmercantilizar y los defensores del $\mathrm{CAE}$, que lo consagraron en la ley" (EI Desconcierto, 2016). La metáfora de la "consagración" del "CAE" en "ley" expresa muy bien aquella complicidad de lo religioso con lo mercantil que encuentra su mediación conservadora en lo legal. La"oscuridad" nos habla de lo invisible que resulta ser esta articulación entre los bloques de poder.

No obstante, en segundo lugar podemos dar cuenta de cómo un discurso masculino hegemónico es puesto en escena. No es casual que la destitución sea hacia la primera mujer rectora de una universidad estatal en Chile. Según Saracostti (2006), en el año 2000, sólo cuatro mujeres se desempeñaban como rectoras en nuestro país (6,25\%), y en 2005 este número ascendió sólo a cinco (8\%).

Según el Informe PNUD de 2015, en una muestra de 20 universidades nacionales de alto ranking, en ninguno de los años estudiados (1995, 2005 y 2009) hubo una mujer ejerciendo como rectora.

Así mismo, Gaete-Quezada (2015) por su parte declara que en 2015 , el $100 \%$ de las universidades estatales tenían un hombre como rector. El gobierno universitario en Chile claramente es masculino no sólo por el hecho de que sean hombres quienes ocupan los altos cargos de dirección, sino además por la discriminación y exclusión constante de las mujeres y la disidencia sexual con respecto al acceso a dichos cargos.

Por último, estamos ante los efectos de un discurso managerial, un paradigma incuestionado en Chile que luego de haberse apoderado de la empresa privada se propagó hacia el ámbito de lo público, siendo conocido entonces como New Public Management (NMP) (Slaughter y Leslie, 1997). Fue durante la dictadura que este modelo logra instalarse y consolidarse como un discurso que permitiría dar respuesta al problema del acceso y la calidad de la educación, creando nuevas universidades-empresas, abriendo sistemas de crédito para costear los estudios, y sobre todo, instalando una lógica de administración del trabajo académico orientada por un modelo capitalista neoliberal. La misma Roxana Pey lo declara al denunciar que a través de su remoción"lo que se quiere es implantar una figura de gobierno universitario medio gerencial" (El Desconcierto, 2016), en donde lo gerencial claramente busca analogar el rol de un rector al de un gerente empresarial.

La palabra gestión opera como un significante flotante (Laclau, 2006), un concepto que puede servir tanto para denunciar este modelo gerencial como para invisibilizarlo. Pey declara que su despido"el Gobierno lo está entendiendo como un asunto menor, administrativo" (Radio U de Chile, 2016).

Entender algo como "administrativo" en este caso implica no sólo invisibilizar el paradigma del management que subyace y explica este acto de exclusión, sino además intenta despojar de cualquier fundamento ideológico o político a la decisión tomada. El management ó gestión entonces aparece como un modo incuestionado, natural, e invisible desde el cual operan las universidades en Chile (Assaél, et al, 2011) que permite a su vez encubrir diferentes discursos de dominación como el conservadurismo o el patriarcado para que puedan operar de maneras silentes y por tanto eficientes.

\section{¿Qué entendemos por managerial?}

Resulta útil detenerse aquí en un punto, distinguir las distintas manifestaciones de la gestión (managerial) en lo universitario. Hablamos de universidades que se han estructurado en base a un repertorio de discursos y prácticas, en donde el management ocupa un lugar hegemónico al actuar el discurso que encubre con un manto de objetividad, tecnicismo, o imparcialidad las operaciones de 
otros discursos dominantes (Parker, 2002). La gestión, la administración o el management en las universidades chilenas hace alusión a aquellas tareas, procedimientos, demandas, etapas, formalidades y requerimientos cotidianos del quehacer académico. Éstas sostienen a las prácticas de docencia, investigación y extensión con el fin de lograr sus objetivos, y configuran además un campo de acciones, sujetos y áreas organizacionales que van más allá de estas tres prácticas, en donde se juega el control social, económico y político de las universidades (Mandiola et al., forthcoming). El gobierno universitario viene a ser una de sus dimensiones principales.

La gestión visible ó explícita dentro de las universidades se refiere a un conjunto de prácticas ligadas al gobierno universitario lo que ha devenido en un elemento constitutivo de su dimensión política. Las prácticas de gestión institucional configuran una investidura dentro de las dinámicas del poder organizacional formal en las universidades. Se traducen en posiciones desde las cuales se toman decisiones y se ejerce el control organizacional. En este sentido, es posible aventurarnos y aseverar que la especialización de la gestión institucional es paralela a la especialización de las prácticas académicas. Esto configura un escenario donde la política universitaria queda resguardada en los confines de un conjunto de prácticas que formalmente la expresan, mientras el posicionamiento desde las prácticas de docencia, investigación y extensión quedarían despojadas de su posibilidad de incidencia sobre la política institucional.

Este proceso de especialización de la gestión y despolitización de las prácticas académicas es una de las consecuencias que ha traído la predominancia del managerialismo en las universidades chilenas. En lo que concierne a la gestión visible, esto refiere tanto a la emergencia de áreas especializadas en el control y la coordinación de las organizaciones académicas, así como a la creación de roles y figuras de administración directiva (ocupadas muchas veces por los mismxs academicos) que son convocados para tomar decisiones, dar forma a ambientes y culturas de trabajo y enfrentar desafíos organizacionales científica y racionalmente (Conllinson \& Hearn, 2005; Parker, M. 2002).

\section{La universidad managerial}

Ahora bien, entendemos que la práctica del management se desarrolla en articulación con un discurso managerial (Alvesson y Willmott, 1996; Willmott, 2011, Parker, 2002) esto es, una ideología tal como es entendida desde una perspectiva discursiva post-estructural (Laclau, 2006) orientada a la optimización de los recursos y regida por principios de eficiencia y eficacia en pos de la productividad. Productividad que puede generar riqueza para sus 'dueños' o excedentes para reinvertir en la gestión, lo relevante es que el proceso para producir ese superávit es siempre el mismo, a saber optimización, eficiencia y eficacia.

El management recurre al discurso managerial por un lado para constituir las prácticas de investigación, docencia y extensión, regulando concursos de investigación, distribuyendo incentivos a la publicación,jerarquizando indexaciones, cobrando aranceles, publicitando una imagen, demostrando transparencia y sustentabilidad, por ejemplo. Pero por otro lado, coloniza la práctica del gobierno universitario siendo sinónimo de la misma, constituyéndose en la práctica de lo político, teniendo en sus manos las decisiones estratégicas que definen la misión y visión institucionales $y$, con ello, implementando acciones y reformas orientadas a la perpetuación y el perfeccionamiento del managerialismo comotal (Fardella, etal, 2015; Santos-Herceg, 2015; Sistoy Zelaya, 2014).
Todo ello es coherente con las exigencias del llamado capitalismo académico (Slaughter y Leslie, 1997; Fernández, 2011), el paradigma de la universidad-empresa (López y Rodés en Samaniego, 2001) y la avanzada del New Public Management (Carnoy y McEwan en Sisto, 2011, Sisto y Fardella, 2014), siendo esta última una lógica de administración empresarial insertada al ámbito de lo estatal mediante el llamado Consenso de Washington (Gentili, 1998). Dichos sistemas y paradigmas orientan el management en la academia chilena hacia la mercantilización del saber; así mismo organizan la transmisión y construcción del conocimiento desde valores como el individualismo, la competencia, y la rentabilidad, amparados por un manto de neutralidad, objetividad, cuantitativismo, y estandarización.

La gestión entonces, es aquel exterior constitutivo (Laclau, 2006) de la academia chilena, aquello que cuesta verbalizar, sólo se puede mostrar, pero que constituye a la práctica académica y la gobierna. La gestión actúa invisibilizando y naturalizando desde un exterior indecible.

Es por ello que la posibilidad de resistir y transformar la academia chilena implica visibilizar, en primer lugar, a la gestión como práctica dominante o hegemónica, y en segundo lugar, agenciar prácticas académicas articuladas y contestatarias a los discursos hegemónicos desde los que se constituye la gestión. Mencionar por ejemplo, la colaboración, la participación, el énfasis en los procesos más que en los resultados, entre otros posibles significados que puedan orientar el despliegue de nuestro sistema de educación superior.

\section{A modo de conclusión}

Comprendemos entonces, que tanto academia como management son 
diferentes campos de prácticas discursivas entre los cuales se han ido construyendo 'articulaciones' (Laclau y Mouffe, 2011) mediante actuaciones políticas de determinados actores y grupos sociales. Estas articulaciones favorecen la reproducción de un orden masculino hegemónico en la academia chilena, y por tanto excluyen y antagonizan con un conjunto de prácticas y subjetividades situadas al exterior de dicho orden. Por otro lado, el carácter social y político de estas articulaciones ha sido naturalizado e invisibilizado a través de un discurso managerial, lo cual hace necesario reactivar el carácter contingente de aquellas dando espacios a nuevos significados para una práctica que requiere de mayor diversidad, y como su nombre lo indica: universalidad.

\section{Bibliografía}

Alvesson, M. and H. Willmott (1996). Making Sense of Management . London, Sage.

Assaél, J.; Cornejo, R.; González, J.; Redondo, J.; Sánchez, R.; Sobarzo, M. (2011). "La empresa educativa chilena", en Educaçao \& Sociedade. (2011), v. 32, n. 115, p. 305-322.

Bravo, B. (1992), La universidad en la historia de Chile; 1622-1992, Pehuén Editores: Santiago de Chile.

BioBioChile (2.8.16), "Mineduc criticó a Roxana Pey falta de alineación", disponible en http://rbb.cl/elge.

CNN Chile (29 de julio de 2016), Entrevista a Roxana Pey, https://www.youtube. $\mathrm{com} /$ watch?v=9vEw2-70dsk Conllinson \& Hearn, 2005

El Desconcierto (11.8.2016), "Hay personajes muy oscuros operando en la reforma"http://www.eldesconcierto. cl/pais-desconcertado/2016/08/11/ roxana-pey-hay-personajes-muyoscuros-operando
El Mostrador (12 de agosto de 2016), “La Contraloría confirma al Mineduc la remoción de Roxana Pey", http:// www.elmostrador.cl/noticias/ pais/2016/08/12/la-contraloriaconfirma-al-mineduc-la-remocionde-roxana-pey/

Fardella, C.; Sisto, V.; Jiménez, F. (2015) Nosotros los académicos. Narrativas identitarias y autodefinición en la universidadactual.Univ.Psychol.Bogotá, Colombia V. 14 No. 5 PP. 1625-1636

Fernández A., R. (2011) Capitalismo académico en la Educación Superior Chilena ¿Modelo exitoso para el desarrollo de la ciencia? Análisis de las tendencias en la productividad científica en investigación durante la última década, Science for Innovation Año 1-No1, octubre2011, Págs. 3645.

Gaete-Quezada, R. (2015). El techo de cristal en las universidades estatales chilenas, Revista Iberoamericana de Educación Superior, no. 17, vol. 6, pp.

Gentili,P.(1998)."El consenso deWashington y la crisis de la educación en América Latina", Archipiélago, p. 56-65.

Laclau, E. (1990) Nuevas reflexiones sobre la revolución de nuestro tiempo. Nueva Visión, Buenos Aires.

Laclau, E. (2006), Misticismo, retórica y política, Buenos Aires, Fondo de Cultura Económica.

Laclau, E.y Mouffe, Ch. (2011) Hegemonía y estrategia socialista. Hacia una radicalización de la democracia, $3^{\text {ra }}$ edición, $1^{\text {ra }}$ reimpresión, Buenos Aires, Fondo de Cultura Económica.

Mandiola, M., Ríos, N., y Varas, A., (forthcoming) "There is something that we still need to talkabout"Gender constructions and organizational practices in Chilean academia.

Parker, M. (2002), Against Management: organization in the age of managerialism, Cambridge: Polity. PNUD (2010) Género los Desafíos de la Igualdad, p. 229
Radio U. de Chile (2016) http://radio.uchile. cl/2016/08/08/roxana-pey-inquietaver-contradicciones-en-la-reforma-ala-educacion-superior/http://radio. uchile.cl/2016/08/08/roxana-peyinquieta-ver-contradicciones-enla-reforma-a-la-educacion-superior/ Santos-Herceg, J. (2015), Cartografía crítica; el quehacer profesional de la filosofía en Chile, La Cañada, Santiago de Chile.

Saracostti,M.(2006)Mujeresenlaaltadirección de Educación Superior: posibilidades, tensiones y nuevas interrogantes. Revista Calidad en la Educación. Consejo Superior de Educación. Chile Sisto, V. (2011), Nuevo profesionalismo y profesores: una reflexión a partir del análisis de las actuales políticas de 'profesionalización' para la educación en Chile, Signo y pensamiento, Vol. 31, Núm. 59, pp. 178-192.

Sisto, V. y Fardella, C. (2014) El eclipse del profesionalismo en la era de la Rendición de Cuentas. Modelando docentes en el contexto del Nuevo Management Público, Cadernos de Educação, 49, 03-23.

Sisto, V. y Zelaya, V. (2014). La etnografía de dispositivos como herramienta de análisisy el estudio del managerialismo como práctica local. Universitas Psychologica, 12(4), 1345-1354.

Slaughter, Sh.y Leslie, E. (1997) Academic capitalism: Politics, Policies \& the Entrepreneurial University. Baltimore. The John Hopkins University Press. SoyChile.cl ( 27.07.16) http:// www.soychile.cl/Santiago/ Sociedad/2016/07/27/408458/ Rectora-de-la-U-de-Aysen-y-lasolicitud-de-renuncia-La-ministrade-Educacion-no-tiene-la-atribucionpara-lo-que-esta-haciendo.aspx Willmott, H. (2011) Journal list fetishism and the perversion of scholarship: reactivity and the $A B S$ list. Organization 18(4) 429-442. 